REVISTA ANDALUZA DE ANTROPOLOGÍA.

NÚMERO 11: TRABAJO Y CULTURAS DEL TRABAJO EN LA GLOBALIDAD HEGEMÓNICA

SEPTIEMBRE DE 2016

ISSN 174-6796

[pp. 199-221]

http://dx.doi.org/10.12795/RAA.2016.11.09

Recibido: 16/05/2016

Aceptado: 12/07/2016

\title{
ANÁLISIS DE LA IDENTIDAD DE GÉNERO EN RELACIÓN CON EL TRABAJO, EN CONTEXTOS DE CRISIS Y DESEMPLEO EN EL SUR DE LA PROVINCIA DE ALICANTE
}

\section{ANALYSIS OF GENDER IDENTITY IN CONNECTION WITH WORK AND CONTEXT OF CRISIS AND UNEMPLOYMENT IN THE SOUTH OF THE PROVINCE OF ALICANTE}

\author{
ANASTASIA TÉLLEZ \\ Universidad Miguel Hernández de Elche \\ JAVIER ELOY MARTÍNEZ \\ Universidad de Murcia
}

\section{Resumen.}

En este texto reflexionamos sobre cómo ha afectado la actual crisis económica y el elevado índice de desempleo a las identidades de género de hombres y mujeres de una comarca alicantina. Lo hacemos desde un análisis antropológico atendiendo a las representaciones ideológicas hegemónicas existentes sobre género y trabajo y a las repercusiones que a nivel personal, social, cultural e identitario ha tenido el denominado estallido de la burbuja inmobiliaria, especialmente en su población masculina.

Palabras Claves.

Desempleo; género; identidad; crisis económica; masculinidad; antropología 


\begin{abstract}
.
In this text we reflect on how the current economic crisis and the high rate of unemployment influence the men' and women' gender identities in a territorial district of Alicante. We will carry out this reflection beginning from an anthropological analysis which takes into account the existing hegemonic ideological representations on gender and work and the impact that the crash of the housing bubble had on personal, social, cultural and identity level, especially within the male population.
\end{abstract}

\title{
Keywords.
}

Unemployment; gender; identity; economic crisis; masculinity; anthropology

\section{INTRODUCCIÓN}

Este artículo es fruto de una investigación antropológica más amplia que hemos llevado a cabo durante cinco años en el sur de Alicante, concretamente en la denominada comarca de la Vega Baja del Segura, pues ha sido en esta zona donde la crisis y el desempleo ha presentado especial virulencia. Hablamos de un territorio donde los efectos de la crisis económica y financiera que comenzó en 2007 ha afectado de manera sustancial aspectos tan relevantes como: el mercado de trabajo local, el tejido empresarial, el paisaje y sus usos, el nivel de vida de sus habitantes, las estrategias domésticas, la reconfiguración de los tiempos y espacios, el empleo y el desempleo, la economía formal y la sumergida, las relaciones interétnicas y los roles e identidades de género en relación con el trabajo.

Tengamos presente que este territorio, principalmente agrícola hasta los años 70 del pasado siglo, fue configurando su mercado laboral en las últimas décadas en torno al sector inmobiliario asociado al turismo residencial. Estos municipios encontraron en la construcción uno de los sectores más dinámicos debido a la importante dinamización derivada del turismo (especialmente todas aquellas actividades ligadas a la segunda residencia), y por el aumento de edificaciones en áreas urbanas o rurales, que también supuso un estímulo sobre la demanda de mano de obra (Esparcia, 2003: 98). Por ello, con la llegada de la crisis, y al haber basado su economía en la construcción, esta zona puede considerarse un ejemplo paradigmático del fenómeno que muchos denominarán "el estallido de la burbuja inmobiliaria", condenando al desempleo a una parte importante de su población, básicamente masculina.

Hemos de tener en cuenta que desde el inicio de la crisis en el año 2007 han aumentado considerablemente las tasas de paro en toda España. Al finalizar este mismo año era del 8,57\%, alcanzando 23,7\% al terminar el 2014, es decir, elevándose un 15,13\%. En la provincia de Alicante, los datos muestran una tendencia similar, con un aumento del 13,68\%, pasando de un 11,23\% al final del 2007, al 24,91\% cuando acabó el 2014. 
Aunque la Encuesta de Población Activa del INE, no llega al nivel de concreción comarcal, si calculamos la tasa de desempleo entendiendo a la población activa como la suma entre la afiliada a la Seguridad Social y la desempleada, la tasa en la Vega Baja del Segura se elevaría al 30,4\%, frente al $27,5 \%$ que resultaría en la provincia al aplicar esta fórmula, lo cual indica una incidencia del paro todavía mayor. Si nos centramos en los diferentes sectores económicos, y la afectación de la crisis a cada uno de ellos, según los datos del SERVEF vemos cómo el más damnificado fue el de la construcción, donde el número de demandantes de empleo aumentó un 35,4\% en 2007, un 118\% en 2008 y un $140 \%$ en 2009, periodo de tiempo en el que se concentró la caída del sector. Otros como los servicios y la agricultura, también se han visto afectados, pero de una manera más continuada en el tiempo.

Debemos advertir, que en esta comarca de la Vega Baja, la economía sumergida ha sido tradicionalmente muy elevada (Téllez, et al. 2009), por lo que muchas personas al llegar la crisis y perder su trabajo, no están recogidas en los datos oficiales del SERVEF. Especialmente las mujeres dedicadas al aparado del calzado o a los cuidados (servicio doméstico, cuidado de personas dependientes o limpieza del hogar) o lo hombres trabajadores de los sectores de la construcción o de servicios. Ello, junto a la desidia y falta de confianza en la administración pública para ofrecerles un empleo, tras años en paro, hace que debamos considerar que hay muchas más personas que han perdido su trabajo que las que aparecen en los datos de desempleo que las cifras oficiales arrojan.

Es ante esta realidad, y dado nuestro interés en el análisis de las identidades desde la perspectiva de género y su relación con el empleo y el desempleo, como abordamos este estudio. Nos surgían preguntas del tipo: ¿cómo condiciona el estar en paro a las identidades femeninas y masculinas en esta comarca?, ¿está apareciendo un nuevo modelo de masculinidad y feminidad?, ¿será ahora, con este alto índice de desempleo en el sector masculino cuando los hombres se incorporen al trabajo doméstico?, ¿qué significa a nivel personal, social, cultural e identitario ser un/a parado/a y demandar empleo sin encontrarlo?, ¿coinciden los discursos con las prácticas de estas personas y hay alguna diferencia en función del sexo?, ¿se gestiona de modo diferente el tiempo de empleo en base a las representaciones ideológicas hegemónicas y los mandatos de género?

La investigación antropológica en la que se sustenta este texto fue realizada desde 2011 hasta 2015 en los 27 municipios $^{1}$ de la comarca de la Vega Baja del río Segura (Alicante). El principal objetivo ha sido analizar los efectos de la crisis económica

1 Albatera, Algorfa, Almoradí, Benejúzar, Benferri, Benijófar, Bigastro, Callosa de Segura, Catral, Cox, Daya Nueva, Daya Vieja, Dolores, Formentera del Segura, Granja de Rocamora, Guardamar del Segura, Jacarilla, Los Montesinos, Orihuela, Pilar de la Horadada, Rafal, Redován, Rojales, San Fulgencio, San Isidro, San Miguel de Salinas y Torrevieja. 
y el desempleo en la población local desde la perspectiva de género, priorizándose el enfoque cualitativo que nos posibilita el estudio de las representaciones ideáticas de los sujetos sobre el trabajo y el género. Nos interesaba adentrarnos en sus prácticas y en sus ideas, así como en los códigos y significados de los comportamientos de mujeres y hombres de esta zona en torno al mundo del trabajo: doméstico (reproductivo, privado) y extradoméstico (público, asalariado). Nos planteamos, entre otros, los siguientes propósitos: a) comprobar si ante el desempleo prolongado de los hombres hay una nueva división sexual y democratización del trabajo en el ámbito doméstico y de los cuidados, y b) estudiar los efectos del desempleo en la construcción de las identidades de género masculinas y femeninas.

Para su realización, y en base al trabajo de campo etnográfico, se ha priorizadola conjunción de la observación con los datos obtenidos por las fuentes orales, especialmente a través de entrevistas abiertas, entrevistas estructuradas con cuestionario cerrado y grupos de discusión, información que se ha triangulado con las fuentes documentales utilizadas.

Se ha entrevistado a hombres y mujeres de diversos grupos de edad y sectores económicos pertenecientes a la población activa (ocupada o desempleada), al empresariado, a "amas de casa", a jubilados/as, a políticos/as locales y a personal técnico (del SERVEF²), Agentes de Empleo y Desarrollo Local, etc.).

\section{TRABAJOS E IDENTIDAD DE GÉNERO DESDE LAS CIENCIAS SOCIALES}

Consideramos el trabajo como una esfera social donde se genera un producto material e ideático, y donde los y las agentes que intervienen establecen relaciones en las que se manifiestan las divisiones sociales que se dan en la sociedad, tales como las originadas a partir de la diferencia de género, clase social, etnia o edad. La base de estas diferencias estructuran el mercado laboral, y por lo tanto determinan las personas empleadas y las desempleadas. Así, la pertenencia a un grupo u otro como componente fundamental actúa en la estructuración selectiva en el mercado de trabajo.

Diversos estudios han constatado cómo la división sexual del trabajo y las cualidades y destrezas que se adscriben a cada sexo en nuestra sociedad son un constructo cultural fruto de la socialización y no están determinadas de modo innato por el sexo biológico (Téllez, 2001, 2002, 2011; Téllez y Martínez Guirao, 2007, 2009; Martínez Guirao, 2015).

Como ya advirtiera Comas el trabajo es una actividad propiamente humana porque sólo los humanos inscriben las actividades de subsistencia en un marco social y simbólico que amplía las capacidades individuales y las dota de capacidad transformadora (1995: 33-34). Efectivamente, el trabajo que hombres y mujeres desarrollamos no sólo produce bienes y servicios, y genera interacción social sino que se carga de aspectos simbólicos y representaciones ideológicas. Además:

2. Servicio Valenciano de Empleo y Ocupación de la Conselleria de Economía Sostenible, Sectores Productivos, Comercio y Trabajo de la Generalitat Valenciana http://www.servef.gva.es/ 
"Es mediante el trabajo como los sujetos de una comunidad adquieren un reconocimiento determinado, un status y prestigio, se les permite participar en el ámbito de lo compartido socialmente y se les otorga unos rasgos definidores de su propia identidad. Y es que los grupos humanos, definen una parte de su identidad a través del trabajo, porque por muy instrumentales o impersonales que puedan ser las tareas que se realicen, para el trabajador se trata de una experiencia personal, de una forma de relacionarse con la realidad en que vive, de identificarse y de ser identificado" (Téllez, 2001).

Junto a los aspectos señalados, autoras como Jahoda (1987) afirman que el empleo ${ }^{3}$ cumple también la necesidad de estructurar la jornada diaria, favorecer las relaciones sociales, vincular las metas personales con objetivos colectivos e imponer una obligación de realización diaria. Es por ello por lo que compartimos las afirmaciones de Palenzuela, cuando defiende que el trabajo es un elemento central de la vida social, pues no sólo contribuye, desde su aportación material, a la existencia del grupo cubriendo las necesidades socialmente determinadas (de producción y reproducción) y a garantizar los modelos de acumulación, sino que además, desde las formas que adopta su representación ideática y simbólica aporta argumentos de legitimación de la estructura social, cooperando decisivamente a su reproducción (1996: 11-18).

Las situaciones de crisis económicas, por sus consecuencias en el incremento del desempleo y la privación del ámbito laboral, tendrán necesariamente incidencias culturales, sociales e identitarias. Es por ello, por lo que en la última década, y desde la llegada de la crisis económica de 2007, numerosos investigadores desde diferentes disciplinas académicas han puesto su interés científico en el estudio del trabajo y el desempleo (Blanch y Cantera, 2009; Téllez, 2011; Montes y Louzán, 2013; López-Andreu, 2014; Rodríguez, 2014; Sanchis, 2008, 2014; Aguado, 2015). Algunos de ellos, advierten que asistimos a un cambio por parte de la población masculina más joven sobre la propia consideración del trabajo, que lejos del modelo clásico, que aún persiste, valoran, por encima de otros aspectos, el valor instrumental debido a la actual inestabilidad laboral del mercado de trabajo en contextos de crisis (Borrás et al, 2012: 419).

Por otro lado, las categorías culturales del género son fundamentales para descubrir la relación entre las distintas funciones asignadas a hombres y mujeres en los ámbitos de la producción y de la reproducción social en cada momento histórico (Aguilar, 1993), y es a partir de dicha relación, como se elaboran las premisas culturales que definen a ambos géneros en nuestra cultura. Porque el género es un elemento estructurante de la realidad, y presente en otros ámbitos de la misma, puesto que define las relaciones entre los seres humanos asignándoles diversos papeles que, al ser construidos como desiguales, sitúan a hombres y mujeres en distintas posiciones (Téllez, 2001).

3. Adviértase que en este texto cuando hablamos de "trabajo" no sólo nos estamos refiriendo al empleo (remunerado) sino que incluimos bajo ese término también al denominado trabajo doméstico o del hogar, aunque no todos los autores y autoras así lo entiendan como el caso Jahoda en esta cita. 
Las categorías de género actuarán en todas las realidades sociales de los sujetos, y por lo tanto en el mundo de la producción laboral, es decir, en el mundo del trabajo productivo y el reproductivo, con sus respectivos espacios y tiempos feminizados y/o masculinizados y el desigual valor que a ambos se les da. Se hace preciso pues profundizar en el nivel de lo ideático, y su función y protagonismo a la hora de guiar, reproducir y justificar las prácticas de los actores sociales en relación con el trabajo y con la ausencia de éste en sentido amplio en función del género.

Téngase presente que uno de los grandes acontecimientos que han caracterizado la segunda mitad del siglo XX en nuestro país ha sido la incorporación masiva de las mujeres a la actividad laboral. Esto supuso una profunda transformación que ha afectado al status de la mujer en su identidad, su feminidad, su independencia, su libertad, sus tiempos, sus espacios y su interacción social, entre otros muchos aspectos. Pero esta incorporación femenina al mundo laboral no se vio correspondida en igualdad por la incorporación de los hombres al mundo de lo doméstico y de los cuidados (Comas, 2015; Carrasco, et al, 2011).

La mujer se incorpora al mercado de trabajo desde un espacio de distribución de roles totalmente consolidado en una cultura androcéntrica y machista, en la cual ella cumple una función reproductiva y de soporte familiar. Así, su identidad femenina no se sustenta sobre el pilar del trabajo remunerado, sino sobre otros pilares (la casa, la maternidad, etc.), por ello, la situación de estar desempleada puede que le afecte de modo diferente al del hombre. Pues sobre el hombre recae la función productiva y se convierte en el sostén económico de la familia. Esta distribución de estereotipos establecidos en función del sexo y justificada, reproducida y mantenida por las representaciones ideológicas de género y trabajo hegemónicas, tienen un peso importantísimo en la perpetuación de la división del trabajo entre mujeres y hombres, tanto en el ámbito familiar como en el laboral y en la sociedad en general. Estas representaciones ideáticas consagran, así, un círculo cerrado de obstáculos que impiden la consecución de la igualdad real de género, convirtiéndose en uno de los factores que más influencia ha tenido y tiene en la configuración de la situación discriminatoria de la mujer en el terreno laboral.

Con respecto a la identidad masculina sabemos, por numerosos estudios, que se construye en gran medida asociada a actividades desarrolladas en el ámbito de lo público, es decir, espacios sociales más allá del ámbito privado-doméstico donde los hombres pueden demostrar su masculinidad, teniendo presente que lo de "demostrar" es condición sine qua non para la identidad masculina. Entre todos estos espacios "públicos" masculinos (espacios de la cultura, la política, el deporte, la educación...), en oposición a los espacios del hogar y de los cuidados, reservados a mujeres, el mundo del trabajo tiene un puesto prioritario. Como se ha advertido (Sanfélix y Téllez, 2014: 387), en todos estos espacios, incluido el del trabajo, competir y mostrar sus logros forma parte sustancial 
de la construcción de este tipo de masculinidad hegemónica. Así, sus logros en la vida no tienen que ver con la parte emocional, ni con sus relaciones personales, sino más bien con aquello conseguido y demostrado, material o simbólicamente en el espacio público, donde el reconocimiento de los iguales y de la misma sociedad reafirma un ideal de masculinidad del que ni siquiera parecen conscientes. Los hombres pues, lo son, en función de su trabajo asalariado, se definen como tales en relación con sus ingresos, lo que les permite cumplir con la principal función asignada socialmente: la proveedora (Gilmore, 1994; Rodríguez, 2014). Sus vidas, en tanto y cuanto hombres, podrían quedar delimitada entre el workaholismo (adictos obsesivos al trabajo) y el concepto clásico de breadwinner (ganapán, proveedor, que mantiene a su mujer, hijos e hijas) (Sanfélix y Téllez, 2014: 388), y ello está determinado por las representaciones ideológicas hegemónicas que existen en nuestra cultura sobre género y trabajo.

Cuando hablamos de representaciones ideológicas o sistemas de ideas y creencias nos referimos a un conjunto coherente internamente de símbolos, valores, nociones e institucionesasociadas queproponenunavisión del mundoyun códigodecomportamiento y que inducen a sentimientos y motivaciones culturalmente compartidas por un grupo humano. Las representaciones ideáticas de género se transmiten de generación en generación y se sustentan en procesos de socialización diferentes en función del sexo de las personas. Así podemos afirmar que:

"La socialización diferencial tradicional ha llevado a que hombres y mujeres adoptaran comportamientos diferentes y desarrollaran su actividad en ámbitos diferentes. Y estas diferencias entre hombres y mujeres (generadas por la socialización diferencial) han contribuido a confirmar la creencia de que son diferentes y se comportan de forma diferente y a justificar la necesidad de continuar socializándolos/as de forma diferente. Es decir, la socialización diferencial es un proceso que se auto-justifica a sí mismo, con todo lo que ello supone" (Ferrer y Bosch, 2013: 108).

Por otra parte, son numerosos los estudios que han puesto de relieve las diferencias estructurales entre mujeres y hombres en cuanto a la experiencia del tiempo, su percepción y su uso, y de manera preferente referido al tiempo de trabajo y "de no trabajo" (Álvaro, 1996; Alfonso y Poeschl, 2006; Amoroso et al., 2003; Borrás, et al, 2007; Callejo, 2005; Carrasco et al, 2003; Carrasco, 2009), de modo que su disposición o carencia en relación con las necesidades personales se ve afectada irremediablemente por la variable género. Estos estudios, entre otros, perfilan una experiencia femenina en la que el "tiempo para los demás" forma parte de las propias expectativas vitales, en contradicción muchas veces con las necesidades de empleo remunerado, independencia y autonomía, frente a la identidad masculina que inculcaría un significado del tiempo en función de un proyecto individual, en el que las expectativas vendrían marcadas por las propias necesidades y deseos, realizadas en el ámbito de lo público. 


\section{EFECTOS DEL DESEMPLEO EN HOMBRES Y MUJERES CON PERSPECTIVA DE GÉNERO}

Como advierten desde la psicología autores como Montes y Louzán (2013: 29) las repercusiones individuales del desempleo comenzaron con las grandes crisis financieras del siglo XX analizando los efectos adversos sobre la calidad de vida y la salud (Eisenberg y Lazarsfeld, 1938; Lazarsfeld, et al, 1996); y se acentuaron a partir de la década de 1970 más centrados en la experiencia individual del desempleo tanto en Europa como en España (Álvaro, 1992; Blanch, 1990, 2001; Garrido, 1999; De Cuyper, et al, 2005; Piqueras, et al, 2008). Y este tema cobra especial atención de nuevo con la actual crisis económica y financiera (Catalano, 2009; Labonte, 2009). Así:

"Ha sido ampliamente reconocido y documentado que el desempleo es un acontecimiento estresante que se traduce en una experiencia psicológica negativa (ver McKee-Ryan, Song, Wanberg y Kinicki, 2005; Paul y Moser, 2009). En comparación con las personas que conservan su empleo, los desempleados manifiestan, entre muchos otros síntomas, mayor sintomatología depresiva (Bolton y Oatley, 1987), mayor sintomatología ansiógena (Layton, 1987), mayor sintomatología psicosomática (Viinamäki, Koskela y Niskanen, 1993), mayor afectividad negativa (Kirchler, 1985), menor autoeficacia (Lang y Lee, 2005), menor calidad de vida (Del Pozo, Ruiz, Pardo y San Martín, 2002), mayor probabilidad de alcoholismo (Dooley, Catalano y Hough, 1992) e, incluso, mayor probabilidad de comportamientos suicidas (Platt, 1985)" (Montes y Louzán, 2013:29).

Por su parte, sociólogos y antropólogos (Poveda, 2006; Téllez y Martínez Guirao, 2007, 2009; Aguado, 2015) han centrado su interés en la vivencia diferenciada del desempleo en mujeres y hombres analizando desde la óptica de género los factores subyacentes a estas desiguales percepciones y experiencias. Recordemos, como ya apuntara Izquierdo (2013), que el orden social en nuestra sociedad se sustenta en la subordinación en donde el sistema sexo/género no es un orden caracterizado por relaciones de complementariedad sino de desigualdad y de división sexual del trabajo.

A su vez, como afirma Aguado

"Los cambios en la división sexual del trabajo y las dinámicas identitarias de los hombres pueden verse afectadas por las transformaciones acontecidas en el mundo del empleo. Para Merla (2006), la inestabilidad, la precariedad de las trayectorias laborales así como las deficientes condiciones de empleo constituyen factores explicativos del distanciamiento entre identidad y empleo también en los hombres" (Aguado, 2015: 213).

4. En un reciente estudio Montes y Louzán (2013) examinan el impacto del desempleo en el bienestar psicológico de las personas que lo han padecido como consecuencia de la crisis económica española actual, centrándose en el sector de la construcción, y atendiendo al tipo de contrato (contrato temporal, autónomos, contrato indefinido) o a su ausencia. 


\subsection{Cambios en la interacción social}

Uno de los efectos que más hemos observado y más se ha repetido en las entrevistas ha sido el cómo el desempleo ha afectado negativamente a las relaciones de pareja y al entorno familiar de nuestros informantes.

"Pues ¡ni te cuento!... yo he estado en paro dos años seguidos...y ahora él lleva ya tres...Y todo son quejas...y se deprime...y está de mal humor desde que se levanta hasta que se acuesta... Yo lo entiendo...pero lo sufrimos su hija y yo...y su padres también...jbueno! Creo que empezando por él...lo sufre toda la familia... Está mal y ya no es el mismo..." (Mujer, empleada, sector servicios, 34 años, Albatera)

En relación con la anterior cita queremos precisar que este conflicto es más evidente en el caso en el que sea el hombre el desempleado en la unidad doméstica. Efectivamente, como nuestro trabajo de campo nos ha puesto de relieve, la construcción cultural de la masculinidad tradicional y hegemónica está muy presente en estas situaciones problemáticas familiares. Aún a sabiendas de que éste es un tema muy complejo, qué duda cabe de que el malestar dentro de la pareja y del entorno familiar más amplio, basado en la situación prolongada de desempleo y falta de ingresos, así como por la "desesperante" situación, sobre todo del varón, de "estar en paro mucho tiempo" incrementa directamente los problemas conyugales, desembocando, en ocasiones, en episodios de violencia intrafamiliar, de separaciones, de estrés y de múltiples formas de "disconfort". Como expertas como Cucco han evidenciado, el desempleo en estos hombres con mentalidad patriarcal les sumerge en una situación de malestar, al verse privados de un elemento clave de identificación, el rol de proveedor, que hace que "en la vida cotidiana normal está presente la queja, queja que denuncia malestares" (2006: 65). $\mathrm{Y}$ es especialmente importante considerar "todos aquellos malestares que la población sufre y que habitualmente no analiza, ni cuestiona porque los considera normales, que no generan demanda explícita, sino que ocupan el lugar de la queja, que no tiene interlocutor válido y que sin embargo se cobran altos precios en Salud y Bienestar" (Cucco y Losada, 2002: 32).

Del mismo modo, en el trabajo de campo, se ha desvelado cómo un gran número de estas personas desempleadas, de nuevo principalmente hombres, van aislándose cada vez más y ven reducir su círculo de amistades. Y es que a menudo comprobábamos en numerosas entrevistas cómo, una cantidad significativa de hombres entrevistados, de mediana edad, que llevaban un tiempo prolongado sin empleo, admitían su desolación y apatía, su falta de confianza en sí mismo y su soledad. Reconocían que no tenían ganas de "dar explicaciones" sobre su situación, ni de "escuchar consejos sobre cómo encontrar trabajo", por lo que optaban por no relacionarse con los vecinos, conocidos, amigos, e incluso, familiares. 
Además, al no trabajar, obviamente ya no interactúan como antes con los/as compañeros/ as de trabajo, y pierden redes sociales, que podrían ofertarles quizás, oportunidades de obtener empleo. Pero a su vez, inmersos en su continua queja, estado de apatía y frustración, prefieren recluirse en su ámbito familiar más cercano y dejan de acudir a otros espacios de sociabilidad, quizás por "vergüenza", como nos afirmaban algunos, o "por tener que reducir gastos", según otros.

Al respecto, hemos comprobado en familias de reducido ingreso y desempleo que ya no se celebran los cumpleaños, las bodas, lo bautizos, como antes de la crisis en la comarca...y se buscan excusas de todo tipo para no asistir a estas ceremonias si se es invitado/a. Además, los más jóvenes, nos confesaban, que "ni siquiera tenían ánimo de meterse en redes sociales para buscar empleo" o "colgar cosas en su Facebook o Linkedin".

\subsection{Desempleo y violencias}

Otro de los aspectos más relevantes de nuestro estudio, ha sido constatar las múltiples formas de violencia que se genera en relación con la situación de desempleo. Especialmente en los hombres, se crea todo un entramado de insatisfacciones identitarias, personales, económicas, entre otras, que se exteriorizan en violencia contra sí mismos, contra sus parejas y contra su entorno familiar en general.

A menudo, nos relataban nuestros/as entrevistados/as experiencias propias o cercanas de violencia verbal e incluso física dentro de hogares donde uno de los dos cónyuges, o los dos, estaban en paro y escaseaban los ingresos. Pero esto no siempre era así, a veces esta violencia intrafamiliar, ejercida en un número de casos importantes por parte del padre de familia desempleado, se podía dar en grupos domésticos donde sí entraba un salario, femenino. En estas ocasiones se ponen en juego todo un conjunto de resortes identitarios y frustraciones del varón relacionadas con la identificación con la masculinidad patriarcal que venimos exponiendo. Veamos lo que comenta una de nuestras informantes sobre su marido:

"Él lleva ya dos años en paro...que no le sale nada de nada...y se encabezona en seguir buscando todos los días...el día lo pasa fuera...va de un pueblo a otro, de una empresa a otra...eso creo al menos yo y él me dice...jClaro! ...Por la noche...no hay quien esté a su lado... ¿ves? Salta a la más mínima... a los chiguitos les grita por todo...está muy, muy alterado... y cuando viene...juf! bueno, ipues eso!.." (Mujer, empleada, sector servicios, 43 años, Cox).

Dentro de los episodios de violencia familiar a los que nos estamos refiriendo, merecen una investigación en profundidad los casos de violencia de género en la pareja. A pesar de que no nos ha sido posible cuantificarlo estadísticamente, si hemos detectado una opinión mayoritaria en los informantes sobre el posible aumento de la violencia machista en estos hogares de la zona analizada. Y siguiendo nuestras reflexiones, consideramos 
que podría existir una conexión entre mentalidad machista en paro y violencia de género contra la mujer.

Al respecto hemos de advertir que a veces se considera erróneamente que la violencia de género ha disminuido, si se atiende a los casos de denuncias de las mujeres, cuando la crisis ha conllevado en muchas situaciones que estas mujeres, denuncien menos los malos tratos, por miedo a no contar con ayudas económicas que les permitan salir de su situación, debido a los recortes y las políticas de austeridad de los últimos años. Por ello, que se reduzcan las denuncias no significa que lo hagan las situaciones de maltrato. Como advierte Alcañiz, (2015: 37) según los datos de la Delegación del Gobierno para la Violencia de Género "el número de denuncias ha disminuido desde el año 2007 pero eso no quiere decir que haya descendido el número de actos violentos sino que probablemente, desde el inicio de la crisis, la situación de muchas mujeres se haya vuelto más vulnerable y consecuentemente desistan de plantearse denunciar por las consecuencias negativas que ello les puede acarrear". Porque en muchas ocasiones, la dependencia económica de estas mujeres de sus maltratadores, se ha visto agravada por la crisis económica y su repercusión en el sector femenino.

Otro de los temas, igualmente muy complejos de analizar, ha sido la relación directa entre las situaciones de desempleo, la masculinidad machista y los casos de suicidio que desde el comienzo de la crisis económica se han dado en esta zona de estudio. Y es que, efectivamente, entendemos que es necesario analizar con perspectiva de género los suicidios en nuestro país, y en la comarca analizada, en relación al desempleo.

Si atendemos a los datos ofrecidos por el INE en los años 2011 y 2012 los suicidios aumentaron en España un 11,3\%. En el 2012, se registraron 3.539 casos, la mayor cifra desde 2005, y que supone 7,5 cada 100.000 habitantes (77\% hombres y $23 \%$ mujeres). En el resto de años desde el 2008 el porcentaje masculino de suicidios siempre es significativamente mayor que el femenino, y esto ocurre en todas las provincias españolas (Massot, 2014), incluida la de Alicante, en la cual en 2012, el 22,4\% de personas que se quitaron la vida fueron mujeres y un $77,6 \%$ de suicidas eran hombres.

Además, hay que detenerse en ver que desde 2008 hasta 2011 (primeros años de crisis) tanto en esta provincia alicantina como en el resto del país, los porcentajes de personas suicidadas eran menores que en el 2011-2012. Concretamente y según nos indica el INE (2014), y analizando la evolución de las tasas de suicidios, en la provincia de Alicante entre los años 2008 y 2012 teniendo presente las defunciones según causa de muerte, la variación de 2008 a 2012 fue de un 23,8\%, mientras que la variación de 2011 a 2012 fue de un $35,3 \%$. La explicación pudiera estar en que es entonces cuando muchas más personas desempleadas por varios años (sobre todo hombres) dejasen de percibir ayudas y prestaciones, encontrándose en situaciones muy difíciles económica y psicológicamente que afrontar. Sobre todo, si tenemos en cuenta que la tasa de paro se incrementó del 11,23\% al finalizar el año 2007 al 25,76\% en 2011, llegando al 28,20\% en 2012. 
En relación con ello, y sabiendo que el tema del suicidio sigue siendo tabú en nuestra sociedad, en varias ocasiones nos encontramos que nuestros informantes nos hablaban del tema, casi “justificándolo" y de modo "muy cercano", relacionándolo directamente con la situación de crisis, de paro y de desahucios de viviendas en la comarca analizada. Ponemos una cita como ejemplo:

“Es que se te pasa de todo por la cabeza! Ya son varios años sin un trabajo por más que busco... y busco todos los días...total ¿para qué? ¡Te frustras tanto!... Y ves que no hay dinero, que no se llega, que pides a tu familia, a tus amigos, que dejan de querer verte... ¡ni tú los quieres ver a ellos!... Vergüenza...mucha. Se te van las ganas de vivir...No sé...pero en los dos último años yo conocía a dos hombres que se los encontraron a uno ahorcado y al otro ¡no se sabe bien!..." (Hombre, desempleado, sector de la construcción, 45 años, Orihuela) Según los datos del INE (2014) la provincia de Alicante triplicó en 2012 la subida porcentual de suicidios registrada en el resto del país, pues en ese año se quitaron la vida 161 personas en esta provincia, un 35,3\% más que en 2011 que fueron 119. De esas 161 personas suicidadas en 2012, 36 eran mujeres frente a 125 que eran hombres. Hacía 20 años que no se daban esas cifras de suicidios en esta provincia según el INE.

Volviendo a la anterior cita de nuestro informante, y tras las cifras ofrecidas por las fuentes estadísticas, y a sabiendas de lo difícil que resulta cuantificar la realidad de los suicidios y sus causas, sí consideramos oportuno apuntar una relación directa, al menos en muchos casos, de las representaciones ideológicas hegemónicas que existen en nuestra cultura que hacen que los hombres sufran una mayor presión social e identitaria si se encuentran en paro "por mucho tiempo". Los datos cuantitativos también apuntan en ese sentido, con el similar incremento entre hombres y mujeres de las tasas de paro en la provincia desde el inicio de la crisis ${ }^{5}$.

Para un porcentaje muy elevado de hombres analizados, ser un desempleado de larga duración supone, por un lado, no ingresar dinero en el grupo familiar, y por lo tanto dejar de ser el mantenedor económico del mismo, el breadwinner o "ganapán”... Por otra parte, representa un fracaso de cara al resto de personas de su entorno social, que comparten estas representaciones tradicionales machistas sobre género y trabajo, para quienes un hombre que no trabaja, estando en edad para ello y siendo su principal obligación, será un "fracasado", un "menos hombre" y aún peor, "un mantenido" (en muchos casos por su mujer), lo que cercena en la misma base de su "hombría" su proyecto no sólo laboral sino vital e identitario.

5. Al finalizar el año 2007 la tasa de paro en la provincia estaban en el 10,2\% para los hombres y el $12,7 \%$ para las mujeres, y al finales de 2014 había subido a $23,9 \%$ para ellos y al $26,1 \%$ para las mujeres. Esto supone unos incrementos del 12,7\% y del 13,4\% respectivamente (INE, 2015). 


\subsection{Efectos en la salud}

En esta investigación hemos podido constatar también, al igual que han demostrado otros investigadores en estudios recientes (López-Adreu, 2014; Sanchis, 2014), que una prolongada situación de inactividad laboral forzada tiene consecuencias directas en la salud (depresión, ansiedad, dolores musculares, angustia e ideas suicidas).

"Desde que estoy en paro, ¡y ya han pasado cinco años desde que cerraron mi empresa!, me han salido miles de achaques (la vista, se me cae más el pelo, picores...me duele todo...Yo nunca antes había tenido que ir tanto al médico...pero es que ahora... ahora... ¡no sé!...Mi mujer dice que es ansiedad y que me sale por todos lados... que mi cuerpo lo nota. ;Yo no lo sé!...pero siempre estoy cansado...y con malos humos...Si te digo, más cansado que cuando iba a la fábrica cada día... ¿te lo explicas?... Me duele mucho la cabeza...quizás de darle tantas vueltas..." (Hombre, desempleado, sector industrial, 57 años, Benijófar)

Otras investigaciones (Warr, et al. 1982, Del Pozo et al, 2002; Selenko et al, 2011, Montes y Louzán, 2013) demuestran que el bienestar psicológico de las personas desempleadas suele decrecer conforme aumenta el tiempo en el que están sin trabajo, tal y como se confirma en nuestra investigación.

"Al principio, pues... iya ves!...te vas haciendo a la idea...y ves la parte positiva de estar en paro...no te levantas a las 6:30 de la mañana para ir a la obra...Pero va pasando el tiempo...y no te encuentras...no te ves bien...y te sientes cada vez peor... y solo...te falta algo... algo diario si no vas a trabajar...Estás... ipues eso! agobiado por el dinero y por otras cosas... No sé...va pasando un año y otro...y...te vas perdiendo... ¡Yo quiero trabajar!...lo necesito..." (Hombre, desempleado, sector de la construcción, 52 años, Rojales)

Junto a la duración de ese estar en paro hay que considerar las condiciones en las que se realizan ciertos trabajos, pues también guardan una estrecha relación con la salud. Es destacable la conexión entre empleos temporales o precarios y determinadas enfermedades mentales y el propio bienestar personal (Agulló, 2001; Amable et al., 2001). "Antes de la crisis sí estaba mejor mi trabajo...la cosa se ha puesto peor... ¡Pues ahora trabajando ocho y diez horas, dependiendo del día, y cotizando tres!...Y con mucha más faena y más estrés...menos tiempo de descanso...Y si a eso le sumas que el dueño de la fábrica nos paga cuando puede o cuando quiere...pues jimagínate que desespere!.. Y ¡a callar!... ¿eh? 'Que como tú hay muchos deseando este trabajo'... Así no se puede aguantar mucho..." (Hombre, empleado, sector industrial, 61 años, Rafal)

"Si es que la mitad del pueblo no tiene trabajo...y de la otra mitad la mayoría sin contrato o a tiempo parcial... iy dan gracias los que lo tienen!... ique lo suyo cuesta como está la cosa!.. Yo sólo trabajo cuatro horas diarias...y no me quejo...me viene bien con mis dos chiguitos pequeños y mi marido yendo de un lado para otro todos los días" (Mujer, empleada, sector servicio, 36 años, Catral) 
Analizando la anterior cita y con respecto a las personas que tienen un contrato temporal habría que diferenciar entre quienes aceptan voluntariamente este tipo de contrato y quienes se ven obligados a ello. Un estudio de Blanch y Cantera (2009) demuestra que si la temporalidad es voluntaria el bienestar psicológico de los trabajadores es parecida a la de los que tienen un empleo estable, frente a los que desempeñan un trabajo temporal involuntariamente los cuales llegan en ocasiones a tener menos bienestar que los desempleados. Ni que decir tiene, la división sexual del trabajo y los mandatos de género hegemónicos juegan en hombres y mujeres un papel decisivo a la hora de percibir la voluntariedad de la temporalidad, eventualidad o intermitencia de su empleo, y por supuesto, del desempleo.

En base a la observación directa de nuestro trabajo de campo y a la información obtenida en un gran número de entrevistas, hemos de resaltar que otro de los efectos de la crisis y el desempleo en esta comarca es el aumento de casos de alcoholismo ${ }^{6}$.

Como se ha comprobado, a través de las entrevistas y con la observación directa, muchos hombres de la comarca, en edad de trabajar, que se encuentran desempleados, están en bares y terrazas a lo largo de la mañana. A horas en las que normalmente hace unos años no se les veían ahora ocupan nuevos espacios urbanos y hacen uso de lugares "masculinos" donde relacionarse entre ellos. Para algunos de nuestros entrevistados frecuentar asiduamente el bar, en ocasiones, varias veces al día, hace que beban más alcohol lo que junto al estado depresivo y pesimista en el que muchos "padres de familia" se encuentran conlleva problemas de alcoholemia con sus respectivas consecuencias en la salud y en el entorno familiar, especialmente, en deterioro de la relación de pareja.

"Sí, voy al bar...ahora más que antes...Desde que estoy en paro...es donde paso más tiempo...¿qué voy a hacer si no?... En la casa no puedo estar...no puedo...entre cuatro paredes no puedo... En el bar hablo con otros y a veces me sale alguna chapuza: alicatar un cuarto de baño, hacer un murete, cambiar una bañera...sale algo de trabajo... Para eso te tienes que enterar y te tienen que ver que buscas faena... Voy al bar..." (Hombre, desempleado, sector construcción, 57 años, Almoradí)

Con respecto a la cita anterior, queremos comentar algunas cuestiones. Por un lado, cómo el bar es un espacio de sociabilidad para estos hombres, donde se establecen y mantienen relaciones sociales, y se entra en redes informales de empleo que permiten bien acceder a puestos de trabajo, bien ofertar sus servicios profesionales. Esta posibilidad les mantiene de algún modo la esperanza en situaciones difíciles, a la vez que retroalimenta la asistencia a estos espacios de ocio. Por otro, se ha observado cómo en muchas de nuestras entrevistas se explicitaba esta idea de que los hombres necesitaban "salir de casa”...estar fuera, en la calle, en el espacio público "masculino". Para ellos, es de suma importancia

6. Al respecto nos parece muy interesante los estudios realizados por Castejón y Mora (2004) y Martínez (2002). 
"ser visibles" de cara a sus iguales, porque aun estando desempleados, "quedarse en casa es cosa de mujeres"7, lo cual pondría en tela de juicio su identidad como varones.

Como los expertos advierten (Castejón y Mora, 2004) en nuestra sociedad, y en base a las representaciones ideológicas de género y salud, los hombres, casi nunca reconocen su malestar psicológico o su estado de ansiedad o depresión. Pues entienden que no es de hombres el ser débil, deprimirse, no saber afrontar los problemas, los contratiempos, pues lo ven como signos de vulnerabilidad que ponen en entredicho por mandato de género su fortaleza masculina. Esta relación tan nefasta de cuerpo y masculinidad hace que, muchos de estos desempleados que hemos analizado, acudan antes a evadirse a través del alcohol y los amigos en el bar, como hemos comentado, que a ir a la consulta del médico de cabecera o de un psicólogo o psiquiatra.

"Llevo tanto tiempo en paro...que ya casi me hago la idea de quedarme así... Yo nunca he estado tanto tiempo parado (...) Sí, estoy ya muy hastiao y desencantao... ya me ves...jY es que yo tampoco pensaba estar tanto tiempo así!...parao. A veces he estao hasta un año en paro en la fábrica en la que trabajaba...pero luego volvía a trabajar...y me apañaba mientras...Con los 420 euros que he estado cobrando...con la ayuda nada más...no se llega...no da...Esto es muy frustrante...el no trabajar..." (Hombre, desempleado, sector industria, San Fulgencio, 54 años)

Al contrario, sus mujeres pueden admitir más fácilmente, como han evidenciado en sus discursos, que están tomando cierta medicación para el insomnio, estrés o depresión, mientras ellos, sus maridos, en el caso de que se mediquen y tengan su tratamiento psiquiátrico, difícilmente lo reconocerán en público, incluso lo considerarán como algo vergonzoso y que les debilita su pretendida masculinidad (debilidad al fin y al cabo ante las adversidades).

" Ya te digo! Ahora no encuentro trabajo... iy me hace falta tanto como el comer!...Me deprimo mucho... y tengo insomnio y mucha, muchísima ansiedad...Sí, me medico...¿claro! ¿Y quién no?.. Yo, en mi vida, he estado en paro varias veces, $y$ sin quererlo... pero siempre me he buscado la vida. Y antes de esta crisis, aunque haya estado en paro me han salido trabajos...jSí! había... y además...yo buscaba...no me quedaba quieta esperando...buscaba trabajo. Antes era diferente...era mucho más fácil” (Mujer, desempleada, industria, 49 años, Rafal)

Somos de la opinión de que la sociedad patriarcal y machista perjudica tanto a mujeres como a hombres, aunque obviamente sean éstas las que peor parte sellevan. Es importante destacar que "ser hombre es equivalente a estar instalado de golpe en una posición que implica poderes y privilegios, pero también deberes: el privilegio masculino es también una

7. Véanse los trabajos de Driessen (1991) y Frigolé (1978) para profundizar en las relaciones entre identidad masculina y el hecho de "no quedarse en casa" o simplemente salir y "plantarse en su sitio". 
trampa" (Waisblat y Sáenz, 2011: 8). Así, en los casos analizados, en los que es el varón el que se encuentra en desempleo por periodos prolongados, en ocasiones, tienen una postura ambivalente: de un aparte se quejan de la situación, pero de otra, no les gusta "quejarse" o "que les vean como víctimas"...Como apuntan los expertos:

"El hombre, en la apariencia de no tener la carga de los niños y la casa, no tiene peso visible, por tanto es un privilegiado, el hombre es "superior y poderoso", ser trabajador y estar fuera de casa es un privilegio, el hombre no tiene carga. Por tanto si es un privilegiado, no puede denunciar sus malestares, ya que de ese modo "atentaría" contra sus privilegios" (Waisblat y Sáenz, 2011: 8).

\subsection{La "no" democratización de la vida doméstica}

Uno de nuestros objetivos, como expusimos al comienzo del artículo, era comprobar si el desempleo de la población masculina de esta comarca ha conllevado que estos hombres se incorporasen a las tareas domésticas y de los cuidados. Hemos analizado el tiempo de hombres y mujeres lo que nos ha permitido comprobar que aún apenas se da la conciliación de la vida familiar y la vida laboral, desde la corresponsabilidad de los dos sexos en igualdad. Fijándonos en la relación entre el tiempo, la acción y las prácticas de estos sujetos seleccionados a partir de la distribución diferencial que hacen en el uso del tiempo como hombres y mujeres, desde la perspectiva de género, se ha constatado cómo, en la socialización de las mujeres y hombres en el sistema patriarcal respecto al tiempo, se encuentra una de las dificultades para conseguir el objetivo de la igualdad propuesto desde los actuales marcos legales y feministas.

$\mathrm{Y}$ afirmamos esto porque hemos comprobado que sigue existiendo mayoritariamente una discriminación en la construcción del tiempo de las mujeres con respecto a la del tiempo de los hombres, a pesar de encontrarse muchos de ellos desempleados.

"Hasta hace un par de años en mi casa no me ayudaba nadie. Ni mis hijos ni mi marido hacían nada en casa. Cuando él trabajaba, él venía del trabajo, yo ponía la mesa y todo, él no. Ahora, que está en paro, parece que se han concienciado un poco y me ayuda más. Parece que me ayudan los hijos y él más, lo que pasa es que se les olvida y tengo que pedírselo. Hasta hace un par de años todo lo hacía yo" (Mujer, empleada, servicios, 46 años, Orihuela)

Para expertos como Sanchis:

"El paro masculino provoca una reorganización del trabajo doméstico en el sentido de mayor participación del hombre (en el trabajo reproductivo), si bien por lo general el aumento es modesto y la situación sigue estando muy lejos del reparto equilibrado de tareas. En todo caso la posición laboral de la mujer ejerce una gran influencia al respecto, ya que cuando está empleada la participación del hombre es mayor" (2008: 234). 
Aunque es cierto que aparecen casos, aún minoritarios, en los que esta construcción se hace cada vez más igualitaria, sobre todo, en el reparto de tiempo dedicado a las tareas del hogar y a la familia, estos casos de hombres "más igualitarios" son muy pocos representativos.

"Mi marido apenas ha hecho nada durante años... me refiero en mi casa. Pero cuando ya se quedó parado y yo me rompí la cadera, entonces la cosa cambió... Él está mucho más volcado en las tareas domésticas que antes..., aunque yo sigo asumiendo la mayor parte de ellas. Pero no termina de asumir que también es su responsabilidad. Yo hago los desayunos, limpio la casa, preparo las comidas, hago las camas, plancho... él colabora puntualmente en algunas cosas, poner y quitar la mesa, tiende la ropa, va a la compra... En este tiempo está haciendo más que en todos los años de matrimonio anteriores". (Mujer, empleada, servicios, 52 años, Torrevieja).

Ello, depende, en gran medida, de cómo estas personas estén socializadas sobre el tema de la igualdad, y cobra especial interés el cómo socializan, a su vez, a las nuevas generaciones en base a diferentes representaciones sociales e ideológicas con relación a roles de género más igualitarios.

Las mujeres estudiadas, al preguntarles qué es lo que más necesitaban para mejorar su calidad de vida, solían nombrar la falta de dinero, trabajo o estabilidad monetaria, en una proporción menor que los hombres. Sin embargo, la gran mayoría dijeron que necesitaban más tiempo, más ayuda en casa y un mejor reparto doméstico, lo cual resulta muy significativo.

Como hemos comentado anteriormente, muchos son los hombres de la comarca que desempleados pasan varias horas al día en los bares de su localidad. Hay que resaltar, que estos hombres no han cambiado en absoluto su modo de ver y vivir el tiempo masculino y femenino, y lejos, de aprovechar su nueva situación personal e incorporarse al mundo de lo doméstico corresponsablemente, al disponer ahora de todo el tiempo "desocupado", reproducen los roles machistas de género, y "se van de casa" aunque no tengan que "trabajar" (fuera del hogar se entiende), pues no considerarán el trabajo doméstico como trabajo.

Para concluir este apartado, queremos apuntar que hemos constatado cómo los hombres y mujeres investigados, además de socializarse de modo diferente en relación con el uso y valor de sus respectivos tiempos, están a la vez produciendo mediante sus prácticas, el espacio-tiempo del propio mundo de forma "generizada". Esto, nos lleva a profundizar sobre el papel de la construcción cultural del tiempo y el cómo la temporalización está permeada por el sistema de género, para analizar su influencia en la conformación de la identidad de hombres y mujeres, como proceso cultural estructurador. 


\section{REFLEXIONES FINALES}

A través de este estudio hemos podido comprobar, a nivel microsocial, algunas de las consecuencias básicas de la actual crisis económica con respecto a las identidades de género en relación con el trabajo y el constante aumento del desempleo.

La crisis y el paro se manifiestan de diversos modos en estas familias, conllevando cambios en las interacciones sociales, las relaciones conyugales, tensiones en las identidades de género, desencuentros familiares, conflictos en la relaciones intrafamiliares e interfamiliares, etc.

Y nos ha parecido de especial interés, constatar que en ciertos casos en los que la mujer es la sustentadora de la economía familiar se genera en muchos hombres un sentimiento de amenaza a su masculinidad, basada en un sistema de género patriarcal, dando lugar esto a conflictos identitarios y de pareja que propician que, en algunos casos pudieran, derivar incluso en situaciones de violencia de género (física, psicológica y simbólica).

Se ha comprobado que aún es muy minoritario el porcentaje de hombres de esta comarca que se corresponsabilizan con el trabajo en el hogar y los cuidados del ámbito doméstico, y ello, a pesar de que en no pocas familias el hombre se encuentra parado y su mujer es la que trabaja fuera de casa, dada la especial coyuntura del mercado de trabajo local. Así pues, queda aún mucho por conseguir para que exista una igualdad real en el ámbito del reparto del trabajo doméstico y en la democratización de los cuidados.

A esto hay que añadir el efecto sobre la salud de la situación prolongada de desempleo, manifiesta de diversas formas, y con diferencias de género, entre hombres y mujeres en relación con el deterioro físico y psíquico: depresión, ansiedad, dependencia de psicofármacos, alcoholismo, etc.

Pero sobre todo, esta situación de desempleo, en especial el masculino, está incidiendo de cierto modo en contradicciones en la identidad masculina hegemónica hasta este momento. Van surgiendo ambivalencias y diversas masculinidades o aspectos de la masculinidad tradicional que conviven en la misma persona, en el mismo hogar y en la misma familia extensa.

Aunque aún de manera minoritaria, quizás este contexto de desempleo forzoso de los hombres haga que no sólo surjan sino que se consoliden social el ideológicamente nuevos pactos más igualitarios entre los roles sexuales en el ámbito laboral y doméstico, lo que conlleva nuevas reformulaciones identitarias de ambos sexos en relación con el mundo del trabajo y a sus identidades. 


\section{REFERENCIAS BIBLIOGRÁFICAS}

Aguado, E. (2015) “El género del paro. Desempleo y masculinidades”. Arxius, 32, pp.207224.

Aguilar, E. (1993) “Trabajo e ideología sobre el género en la producción doméstica”. En C. Diez. y V. Maqueira (eds.) Sistema de Género y construcción (deconstrucción) de la desigualdad. Tenerife: Asociación Canaria de Antropología, pp. 81-96.

Agulló, E. (2001) "Entre la precariedad laboral y la exclusión social: los otros trabajos, los otros trabajadores". En E. Agulló y A. Ovejero (Coords.) Trabajo, individuo y sociedad: perspectivas psicosociológicas sobre el futuro del trabajo. Madrid: Pirámide, pp. 95-144.

Alcañiz, M. (2015) “Sociología de la(s) violencia(s) de género en España. Una propuesta de análisis”. Revista de Paz y Conflictos 8 (2) http://revistaseug.ugr.es/index.php/revpaz/ article/view/3054 [Consultado el 15 de abril de 2016].

Alfonso, R.Ma y Poeschl, G. (2006) "Representaciones del impacto de la situación de desempleo en las prácticas familiares”. Revista de Psicología Social, 21(3), pp. 241-258.

Álvaro, J.L. (1992) Desempleo y bienestar psicológico. Madrid: Siglo XXI.

Alvaro, M. (1996) "Diferencias cuantitativas y cualitativas, entre mujeres y varones medidas a través de los usos del tiempo”. Revista de Psicología Social, 11(2), pp. 163-184.

Amable, M., Benach, J. y González, S. (2001) "La precariedad laboral y su repercusión sobre la salud: conceptos y resultados preliminares de un estudio multimétodos". Archivos de Prevención de Riesgos Laborales 4, pp. 169-184.

Amoroso, M. I.; Bosch, A.; Carrasco, C.; Fernández, H. y Moreno, N. (2003) Malabaristas de la vida. Mujeres, tiempos y trabajos. Barcelona: Icaria.

Blanch, J.M. (1990) Del viejo al nuevo paro. Un análisis psicológico y social. Barcelona: PPU.

Blanch, J.M. (2001) “Empleo y desempleo, ¿viejos conceptos en nuevos contextos?”. En E. Agulló y A. Ovejero, (coords.) Trabajo, individuo y sociedad: perspectivas psicosociológicas sobre el futuro del trabajo. Madrid: Pirámide, pp. 27-48.

Blanch, J.M. y Cantera, L.M. (2009) "El malestar en el empleo temporal involuntario". Revista de Psicología del Trabajo y de las Organizaciones, 25(1), pp.59-79.

Bolton, W. y Oatley, K. (1987) "A longitudinal study os social support and depression in unemployed men". Psichological Medicine, 17, pp.453-460.

Borrás, V.; Torns, M.T. y Moreno, S. (2007) "Las políticas de conciliación: políticas laborales versus políticas de tiempo”. Papers: revista de sociología, 83, pp. 83-96. 
Borràs, V.; Moreno, S.; Castelló, L. y Grau, A. (2012) "Male hegemony in decline? Reflection of the Spanish case”. Men and Masculinities, 15(4), pp. 406-423.

Callejo, M.J. (2005) "Estrategias temporales: relaciones entre tiempo de trabajo remunerado y tiempo de trabajo doméstico". Cuadernos de Relaciones Laborales, 23(1), pp. 175-204.

Carrasco, C. et al. (2003) Tiempos, trabajos y flexibilidad: una cuestión de género. Madrid: Instituto de la Mujer, Ministerio de Trabajo y Asuntos Sociales.

Carrasco, C. (2009) “Trabajos y tiempos desde la experiencia femenina”. Papeles de relaciones ecosociales y cambio global, 108, pp. 45-54.

Carrasco, C., Borderías, C., y Torns, T. (eds.) (2011) El trabajo de cuidados. Historia, teoría y políticas. Madrid: La Catarata.

Castejón, E. y Mora, G. (2004) Alcoholismo, género y emociones:¡el hombre se alcoholiza y la mujer se deprime? Patrones relacionales en la familia del hombre alcohólico en comparación con los de la mujer depresiva. Barcelona: Ed. Escuela de Terapia Familiar, Hospital de la Santa Cruz, Universidad Autónoma de Barcelona

Catalano, R. (2009) "Health, medical care and economic crisis". The New England Journal of Medicine, 360, pp.749-751.

Comas, D. (1995) Trabajo, género, cultura. Barcelona: Icaria.

Comas, D. (2015) "Los cuidados de larga duración y el cuarto pilar del Estado del bienestar”. Revista de Antropología Social, 24, pp. 173-196.

Cucco, M. y Losada, L. (2002) “Metodología de los Procesos correctores comunitarios”. Rescoldos, 6, pp. 31-36.

De Cuyper, N.; Isaksson, K. y De Witte, H. (2005) Employment contracts and well-being among European workers. Hampshire: Ashgate.

Del Pozo, J.A., Ruiz, M.A.; Pardo, A. y San Martín, R. (2002) “Efectos de la duración del desempleo entre los desempleados". Psicothema, 14, pp.440-443.

Delegación del Gobierno para la Violencia de Género (2016) Portal estadístico del Ministerio de Sanidad, Servicios Sociales e Igualdad. http://www.msssi.gob.es/ organizacion/ministerio/organizacion/SEssi/dgvgF.htm [Consultado el 15 de abril de 2016].

Dooley, D.; Catalano, R. y Hough, R. (1992) "Unemployment and alcohol disorder in 1910 and 1990: Drift versus social causation”. Journal of Occupational and Organizational Psychology, 65, pp.277-290. 
Driessen, H. (1991) "Sociabilidad masculina y rituales de masculinidad en la Andalucía rural”. En AA. VV. Antropología de los pueblos de España. Madrid: Taurus Ediciones, pp.710-718.

Eisenberg, P. y Lazarsfeld, P.F. (1938) “The psychological effects of unemployment". Psychological Bulletin, 35, pp.358- 390.

Esparcia, J. (2003) “Sectores productivos y dinámica socioeconómica. Una aproximación a la situación y cambios recientes en áreas rurales valencianas". Revista Serie Geográfica, 11, pp. 91-115.

Ferrer, V. y Bosch, E. (2013) "Del amor romántico a la violencia de género. Para una coeducación emocional en la agenda educativa". Profesorado. Revista de currículum y formación del profesorado, 17(1), pp. 106-122

Frigolé, J. (1978) "Ser cacique" y "ser hombre": Dos símbolos y dos modelos de relación en un pueblo de la Vega Alta del Segura. En M. Gutiérrez Esteve; J. A. Cid Martínez y A. Carreira (coords.) Homenaje a Julio Caro Baroja. Madrid: Centro de Investigaciones Sociológicas. pp. 371-390.

Garrido, A. (1999) "Psicología Social del Desempleo". En J.L. Álvaro; A. Garrido y J.R. Torregrosa (eds.) Psicología Social Aplicada. Madrid: McGraw-Hill, pp. 121-154.

Gilmore, D. (1994) Hacerse hombre: Concepciones culturales de la masculinidad. Barcelona: Paidós.

INE (2015) Defunciones según la causa de muerte. Suicidios. http://www.ine.es/prensa/ np896.pdf [Consultado el 15 de abril de 2016].

Izquierdo, $\mathrm{M}^{a}$ J. (2013) "La socialización de género". En C. Díaz y S. Moreno (eds.), Sociología y Género. Madrid: Tecnos.

Jahoda, M. (1982) Employment and Unemployment: A Social-psychological analysis. Cambridge: Cambridge University Press.

Jahoda, M. (1987) Empleo y Desempleo: un análisis sociopsicológico. Madrid: Morata.

Kirchler, E. (1985) “Job loss and mood”. Journal of Economic Psychology, 6, p.9-25.

Labonte, R. (2009) “The global financial crisis and health: scaling up our effort". Canadian Journal of Public Health, 100, pp.173-175.

Lang, J.C. y Lee, C.H. (2005) "Identity accumulation, others' acceptance, job search selfefficacy, and stress". Journal of Organizational Behavior, 26, p p.293-312.

Layton, C. (1987) "Levels of state anxiety for males facing redundancy and subsequently reporting to be employed or unemployed”. Perceptual and Motor Skills, 65, pp. 53-54. 
Lazarsfeld, P.F, Zeisel, H., y Jahoda, M. (1996) Los parados de Marienthal: Sociografía de una comunidad golpeada por el desempleo. Madrid: La Piqueta.

López-Andreu, M. (2014) "El desempleo como potencial punto de inflexión. Un análisis biográfico de trayectorias laborales". Sociología del Trabajo, 81, pp.27-49.

Martínez, A. M. (2002) “Alcoholismo femenino: problemática social”. La Ventana, 16, pp. 78-95

Martínez Guirao, J.E. (2015) "Riesgos laborales en la construcción. Un análisis sociocultural”. Universitas, Vol. XIII, NN23, pp. 65-86.

Massot, M. (2014) "Mapa de suicidios desde el inicio de la crisis". http://www.elperiodico. com/es/noticias/sociedad/mapa-suicidios-crisis-provincias-espana-3095090 [Consultado el 12 de abril de 2016]

McKee-Ryan, F., Song, Z., Wanberg, C. y Kinicki, A. (2005) "Psychological and physical well-being during unemployment: a meta-analitic study". Journal of Applied Psychology, 90, pp.53-76.

Montes, C. y Louzán, R. (2013) "Repercusiones psicológicas del desempleo: efectos colaterales de la crisis del sector de la construcción”. Escritos de Psicología, 6(1),pp. 28-35. Palenzuela, P. (1996) "El trabajo, objeto de estudio para la antropología”. Antropología del Trabajo, III Simposio del VII Congreso de Antropología Social, Zaragoza.

Paul, K. I. y Moser, K. (2009) "Unemployment impairs mental health: meta-analyses". Journal of Vocational Behavior, 74, pp.264-282.

Platt, S. (1985) "Suicidal behaviour and unemployment: A literature review". En G. Westcott; P.G. Svensson y H.F.K. Zollner (eds.) Healthpolicy implications of unemployment. Copenhagen, Denmark: World Health Organization, pp. 87-132.

Piqueras, R., Rodríguez, A. y Rueda, C. (2008) "Expectativas y duración del desempleo". Revista de Psicología del Trabajo y de las Organizaciones, 24, pp.129-151.

Poveda, M. M. (2006) “'Los lunes al sol' o 'los lunes en casa'. Roles de género y vivencias del tiempo de desempleo". Cuaderno de Relaciones Laborales, 24(2), pp. 85-110.

Rodríguez, J. A. (2014) “Cuando Cae el Hombre Proveedor. Masculinidad, Desempleo y Malestar Psicosocial en la Familia. Una Metodología para la Búsqueda dela Normalización Afectiva". Masculinities and Social Change, 3 (2), pp. 173-190.

Sanchis, E. (2008) Trabajo y paro en la sociedad postindustrial. Madrid: Consejo Económico y Social (CES).

Sanchis, E. (2014) "El paro hace daño". Sociología del Trabajo, 81, pp. 7-26. 
Sanfélix, J. y Téllez, A. (2014) "Historias de hombres: Recuperando las voces de los hombres reales". Prisma Social, 13, pp. 370-406.

Selenko, E., Batinic, B. y Paul, K. (2011) "Does latent deprivation lead to psychological distress? Investigating Jahoda's model in a four-wave study". Journal of Occupational and Organizational Psychology, 84, pp.723-740.

Téllez, A. (2001) "Trabajo y representaciones ideológicas de género. Propuesta para un posicionamiento analítico desde la antropología cultural". Gazeta de Antropología, 17. http://www.gazeta-antropologia.es/?p=3271 [Consultado el 15 de abril de 2016].

Téllez, A. (2002) "Trabajo, identidad y género: la puesta en juego de las representaciones ideológicas". Cuadernos de Relaciones Laborales, 20(1), pp.191-214.

Téllez, A. (2011) "La jubilación de hombres y mujeres desde la perspectiva de género: un análisis antropológico". Revista Senior. Elche: Ed. Universidad Miguel Hernández de Elche.

Téllez, A. y Martínez Guirao, J. E. (2007) "Mujeres en la industria descentralizada: economía informal y género". En AA.VV. Poder, cultura y civilización. Barcelona: FES y Universidad de Barcelona.

Téllez, A. y Martínez Guirao, J.E. (2009) (eds.) Economía informal y perspectiva de género en contextos de trabajo. Barcelona: Icaria.

Téllez, A. y Verdú, A. D. (2011) "El significado de la masculinidad para el análisis social". Revista Nuevas Tendencias en Antropología, 2, pp. 80-103.

Téllez, A. et al. (2009) Mujer y trabajo en el sector industrial: economía sumergida, violencia y género. Madrid: Instituto de la Mujer.

Viinamaki, H., Koskela, K. y Niskanen, L. (1993) "The impact of unemployment on psychosomatic symptoms and mental well-being". International Journal of Social Psychiatry, 39, pp.266-273.

Waisblat, A. y Sáenz, A. (2011) "La construcción socio-histórica de la existencia. Patriarcado, capitalismo y desigualdades instaladas". Ponencia presentada en Jornadas sobre Roles masculino y femenino a debate, Bilbao.

Warr, P.B., Jackson, P. y Banks, M. (1982) "Duration of unemploymentand psychological well-being in young men and women”. Current Psychological Research, 2, pp. 207-214. 